\title{
Assessment of the Magnetocaloric Effect upon the Magnetic Entropy Change
}

\author{
Ying De ZHANG
}

State Key Laboratory of BaiyunObo Rare Earth Resource Research and Comprehensive Utilization, Baotou Research Institute of Rare Earth - RE High-tech Zone, Baotou, Inner Mongolia, 014030, P.R. China

Tien Van MANH

Department of Physics, Chungbuk National University, Cheongju 361-763, Korea

The Long PHAN

Department of Physics and Oxide Research Center, Hankuk University of Foreign Studies, Yongin 449-791, Korea

Hyeong-Ryeol PARK · Seong-Cho YU*

Department of Physics, Ulsan National Institute of Science and Technology, Ulsan 44919, Korea

(Received 13 October 2020 : revised 05 January 2021 : accepted 06 January 2021)

\begin{abstract}
The magnetocaloric effect is a dynamic phenomenon associated with a temperature change of a magnetic material when it is subjected to a magnetic-field change. The effect can be assessed through the adiabatic temperature change $\left(\Delta T_{a d}\right)$ or the isothermal magnetic-entropy change $\left(\Delta S_{m}\right)$. This work reviews some typical methods that are usually used to calculate $\Delta S_{m}$ for perovskite-type manganites. These methods was thermodynamic relations and different theoretical models to analyze magnetization isotherms, $M(H)$ data, recorded at temperatures around the ferromagnetic-paramagnetic phase transition $\left(T_{C}\right)$, Together with showing the methods for calculating of $\Delta S_{m}$, we also take into account the figures of merit of a MC material.
\end{abstract}

Keywords: Magnetocaloric effect, Magnetic entropy, Analysis techniques

\section{Introduction}

The magnetocaloric effect (MCE) is a dynamic phenomenon related to a temperature $(T)$ change of a magnetic material when it is magnetized or demagnetized, meaning that a magnetic-field change causes a temperature change. This is intrinsic to all magnetic materials and associated with the coupling between magnetic moments and an applied magnetic field $(H)$ [1]. Because the magnetization and demagnetization cycles are similar to isothermal compression and adiabatic expansion processes of gases using in conventional refrigerators, it has been employed the MCE to build magnetic refrigerators [1-3]. It has been found that if comparing with

\footnotetext{
*E-mail: scyu@unist.ac.kr
}

conventional refrigeration, magnetic refrigeration is more environmentally friendly and has higher efficiency cooling. It would allow freezers to liquefy hydrogen or natural gases for use in clean-burning power plants or future automobiles [4]. Such discoveries have rapidly attracted much attention of the scientific and technological community $[2,5,6]$.

In thermodynamics, the temperature change caused by the $\mathrm{H}$ change is tightly related to entropy $(S)$, a parameter is characteristic of the state of disorder of a physical system. The relation between them can be understood through in the S-T diagram, as depicted in Fig. 1 [1, 2]. For a magnetic system, the temperature change taking place in the adiabatic process $\left(\Delta T_{a d}\right)$ or a magneticentropy change in the isothermal process $\left(\Delta S_{m}\right)$ is characteristic of the MCE. High entropy change corre- 


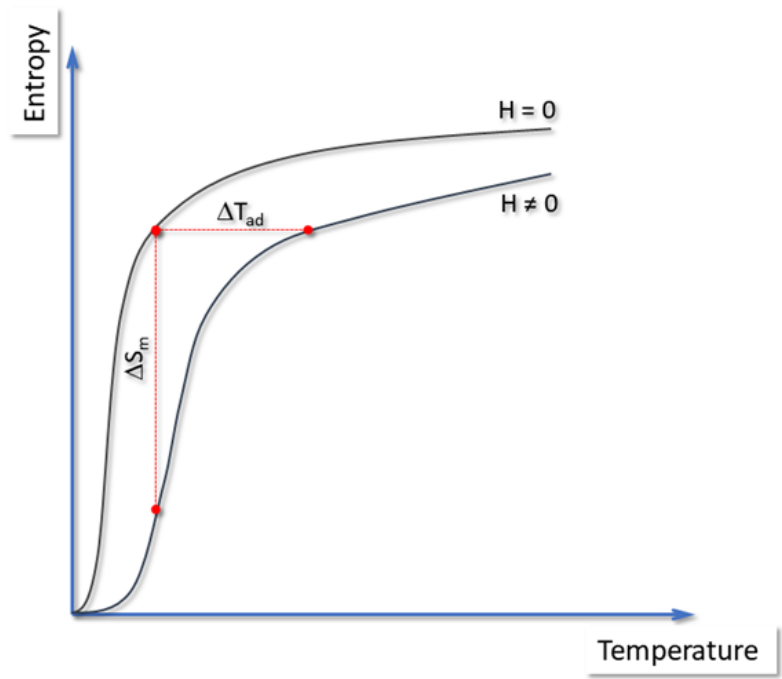

Fig. 1. (Color online) The $S$-T diagram depicted for an $H$ change from $H_{1}=0$ to $H_{2} \neq 0$.

sponds to high disorder of magnetic moments and a large temperature change. This physical phenomenon is usually observed around phase-transition regions; for examples, antiferromagnetic-paramagnetic (AFM$\mathrm{PM}$ ), antiferromagnetic-ferromagnetic (AFM-FM) and ferromagnetic-paramagnetic (FM-PM) transitions [1].

To fabricate conventional cooling systems (air conditioners, refrigerators and chillers) based on the MCE, it would be used magnetic materials showing phase transformations in the temperature range $T=270-310 \mathrm{~K}$. These materials play as coolants in adiabatic demagnetization refrigerators. A good refrigerant ensures the following criteria: (i) a high density of magnetic moments (high concentration of $4 f$ and/or $3 d$ elements), (ii) a strong dependence of the magnetization on $\mathrm{T}$ and $\mathrm{H}$ that ensures a large change in temperature, (iii) a magneticphase transformation occurs around the working temperature, and (iv) high permeability and small magnetic hysteresis to avoid energy losses during the magnetization and demagnetization cycles [7]. A noble and prototypal material used over the room-temperature region is Gd with a second-order phase transition (SOPT) character [8-10]. Apart from Gd, it has also been found potential applications of other magnetic materials, such as rare-earth $(\mathrm{Re})$ intermetallics $\mathrm{ReM}_{2}(\mathrm{M}=\mathrm{Al}, \mathrm{Co}$, and Ni) [11], Gd-Si-Ge [12,13], La/Nd-Fe $\neg-(\mathrm{Si}, \mathrm{H})$ [14-17], Fe-Rh [18], Ni-Mn-(Ga, Sn, In) (Heusler alloys) [19-23], and perovskite-type manganites $\left(\mathrm{Re}_{1-x} \mathrm{M}_{x} \mathrm{MnO}_{3}\right.$, with
$\mathrm{M}=\mathrm{Ca}, \mathrm{Sr}, \mathrm{Ba}$, and $\mathrm{Pb}$ ) [24-29]. However, some potential materials with giant MCE exhibit a first-order magnetostructural or magnetoelastic transition. During cycling, the first-order phase transition (FOPT) reduces the cooling efficiency and the structural change may cause severe damages of refrigerants [7]. Thus, magnetocaloric (MC) materials exhibiting simultaneously both large MCE and SOPT are highly desired in magnetic refrigeration applications.

It should be noticed that as studying a MC material for the refrigeration application, the most important parameter is $\Delta T_{a d}$. Its change versus $\mathrm{H}$ can be directly measured by a sensitive thermocouple attached on the sample [9]. Due to the correlation between $\Delta T_{a d}$ and $\Delta S_{m}$, it can also be assessed both these parameters to gain information about phase transition/separation, magnetic order, coupling mechanisms and so forth $[2,25]$. In some cases, due to a lack of facilities and technical difficulties, one can not perform the direct measurement of $\Delta T_{a d}$. In such cases, it can be indirectly determined $\Delta T_{a d}$ from $\Delta S_{m}$, specific heat capacity $\left(C_{p}\right)$ data, and thermodynamic relations. In experiment, $\Delta S_{m}$ can be obtained from $H$-dependent $C_{p}(T), M(T)$, and isothermal $M(H)$ [30-32]. Additionally, $H$ - and $T$-dependent resistivity, $\rho(T, H)$, data can also be used to calculate $\Delta S_{m}[33]$. This work presents several ways to calculate $\Delta S_{m}$ from the isothermal $M(H)$ data recorded around the FM-PM transition temperature of perovskite manganites. Apart from these contents, the relative cooling power $(R C P)$ and refrigerant capacity $(R C)$ are also taken into account. Herein we use a set of $M(T, H)$ data recorded from $\mathrm{La}_{0.6} \mathrm{Ca}_{0 .} 4 \mathrm{MnO}_{3}$ for $\mathrm{MC}$ analyses and discussion.

\section{Experimental details}

A polycrystalline sample of $\mathrm{La}_{0.6} \mathrm{Ca}_{0.4} \mathrm{MnO}_{3}$ (LCMO) was prepared by conventional solid-state reactions in air. High-purity chemicals $(99.9 \%)$ of $\mathrm{La}_{2} \mathrm{O}_{3}, \mathrm{CaO}$ and $\mathrm{MnCO}_{3}$ in powder with suitable masses, according to the chemical formula of $\mathrm{La}_{0.6} \mathrm{Ca}_{0.4} \mathrm{MnO}_{3}$, were well mixed and ground by mechanical ball milling for $3 \mathrm{~h}$. After that, the powder was pressed into a pellet and annealed at $1320 \mathrm{~K}$ for $12 \mathrm{~h}$. After fabrication, X-ray diffraction 
analysis indicated the LCMO sample exhibiting the orthorhombic single phase (space group: Pnma), with the lattice constants $a=5.432$ Åand $c=7.671 \AA$. Magnetization measurements, $M(T, H)$, were performed on a quantum design physical property measurement system, in which $T$ and $H$ were changed in the ranges of $110-$ $300 \mathrm{~K}$ and $0-60 \mathrm{kOe}$, respectively.

\section{Relations between MCE parameters and assessments}

As mentioned above, the $\mathrm{MC}$ effect is directly assessed through $\Delta T_{a d}$. This parameter is also correlated with $\Delta S_{m}$ and $C_{p}$. According to the second and third laws of thermodynamics, and the fact that measurements can be carried out only above absolute zero, the relation between $\Delta T_{a d}$ and $\Delta S_{m}$ is obtained as follows $[1,34]$ :

$$
\Delta T_{a d}=\frac{T}{C_{p}(T, H)}\left|\Delta S_{m}(T, H)\right|
$$

Here, $C_{p}$ can be considered as the total heat capacity of the electronic $\left(C_{e}\right)$, phonon/lattice $\left(C_{l} a t\right)$ and magnetic $\left(C_{m}\right)$ contributions. For a given magnetic material, depending on its conducting and elastic properties and the investigated temperature range (i.e., the FM, AFM or PM phase), the contribution of $C_{e}, C_{l} a t$ and/or $C_{m}$ will be dominant. From Eq. (1), if knowing $\Delta S_{m}$ and $C_{p}$ data, it will be indirectly obtained $\Delta T_{a d}$. The equation also reflects that to gain large $\Delta T_{a d}$, it is necessary to find a material owning large $\Delta S_{m}$ and small $C_{p}$. Maximum magnetic entropy of a material can be calculated by $S_{\max }=R \ln (2 J+1)$, where $R$ is a universal gas constant and $J$ is the total angular momentum. Thus, a good MC material usually contains transition-metal $(3 d)$ and/or Re $(4 f)$ elements because of large $J$ values.

It should be noticed that from the $C_{p}(T, H)$ data, it can be deduced the total entropy (St) from the following expression:

$$
S_{t}(T, H)=S_{t}\left(T_{0}, H\right)+\int_{T_{0}}^{T} \frac{C_{p}\left(T^{\prime}, H\right)}{T^{\prime}} d T^{\prime}
$$

where the first term is an entropy value extrapolated as $T_{0} \rightarrow 0$. From Eq. (2), absolute $\Delta S_{m}$ (denoted as
$\left|\Delta S_{m}\right|$, associated with the magnetic contribution only) can be calculated by using the expression:

$$
\begin{aligned}
\left|\Delta S_{m}(T, H)\right|= & S\left(T_{0}, H\right)-S\left(T_{0}, 0\right) \\
& +\int_{T_{0}}^{T} \frac{C_{m}(T, H)-C_{m}(T, 0)}{T^{\prime}} d T^{\prime}
\end{aligned}
$$

Alternatively, $\left|\Delta S_{m}\right|$ can be determined from Maxwell's relations as follows:

$$
\left(\frac{\partial S}{\partial H}\right)_{T}=\left(\frac{\partial M}{\partial T}\right)_{H}
$$

$$
\left|\Delta S_{m}(T, H)\right|=\int_{0}^{H}\left(\frac{\partial M}{\partial T}\right)_{H} d H
$$

Apart from these methods to determine $\left|\Delta S_{m}\right|$, one can use other approaches based on theoretical approaches using Landau [35,36], classical PM (Langevin function) [37] and mean-filed [36] theories, and a phenomenological model [38]. We shall present some of these methods that are usually used to assess the MCE of perovskite-type manganites.

\section{IV. $\Delta S_{m}$ calculations based on Maxwell's relations and theoretical models}

The determination of $\left|\Delta S_{m}\right|$ using different theoretical models starts from the $M(H)$ isotherms recorded around the FM-PM transition (the Curie temperature, $T_{C}$ ) of a magnetic material. For example, for a perovskite compound of LCMO used in our current work, after recording its $M(T)$ data and identifying its $T_{C}$ value $(-251$ $\mathrm{K}$, in good agreement with the $T_{C}$ value shown in Ref. [39]), see Fig. 2(a), we measured $M(H)$ isotherms. The feature of the $M(H)$ isotherms can be seen in Fig. 2(b), showing a gradual change in curvature (i.e., a nonlinear $M(H)$ curve becomes linear as increasing $T$ ), due to the FM-PM phase transition. Notably, the $M(H)$ isotherms could be initial magnetization or demagnetization curves. For a soft-magnetic material with small coercivity, the difference between these two data is insignificant. From the set of $M(H)$ isotherms, it is easily calculated $\left|\Delta S_{m}\right|$ using one of the following routes. 

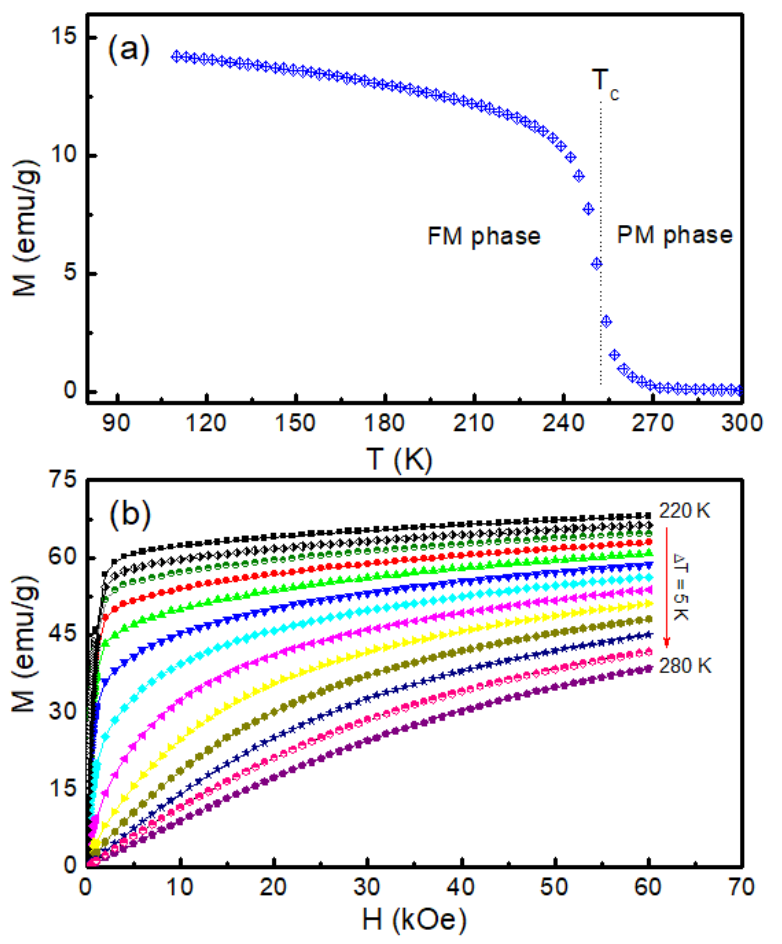

Fig. 2. (Color online) (a) Field-cooled $M(T)$ curve for $H$ $=100$ Oe, and $(\mathrm{b}) M(H)$ isotherms of LCMO recorded around its $T_{C}$ point.

\section{1. $\Delta S_{m}$ calculation using Maxwell's relations}

Among the mentioned methods, the calculation of $\left|\Delta S_{m}\right|$ upon Maxwell's relations, Eqs. (4, and 5), is the most popular [25, 40-42]. Using this method (socalled Maxwell's relations), numerical integration, Eq. (5), gives the result of $\left|\Delta S_{m}\right|$. It should be noticed that Eq. (5) was derived from free energy, thermodynamics requires the equilibrium of the states in the initial zero field $(H=0)$ and the finite field $H$. For a magnetic system undergoing the SOPT, both states satisfy this requirement. Thus, $\left|\Delta S_{m}\right|$ can also be calculated by an approximation formula [34]:

$$
\begin{aligned}
& \left|\Delta S_{m}\left(\frac{T_{1}+T_{2}}{2}\right)\right|= \\
& \quad \frac{1}{T_{2}-T_{1}}\left[\int_{0}^{H} M\left(T_{2}, H\right) d H-\int_{0}^{H} M\left(T_{1}, H\right) d H\right]
\end{aligned}
$$

It appears from Eq. (6) that the isothermal variation of $\left|\Delta S_{m}\right|$ for $\left(T_{1}+T_{2}\right) / 2$ is proportional to the area of the region between two adjacent $M(H)$ curves. To more accurately determine $\left|\Delta S_{m}\right|$, it is necessary to record $M(H)$

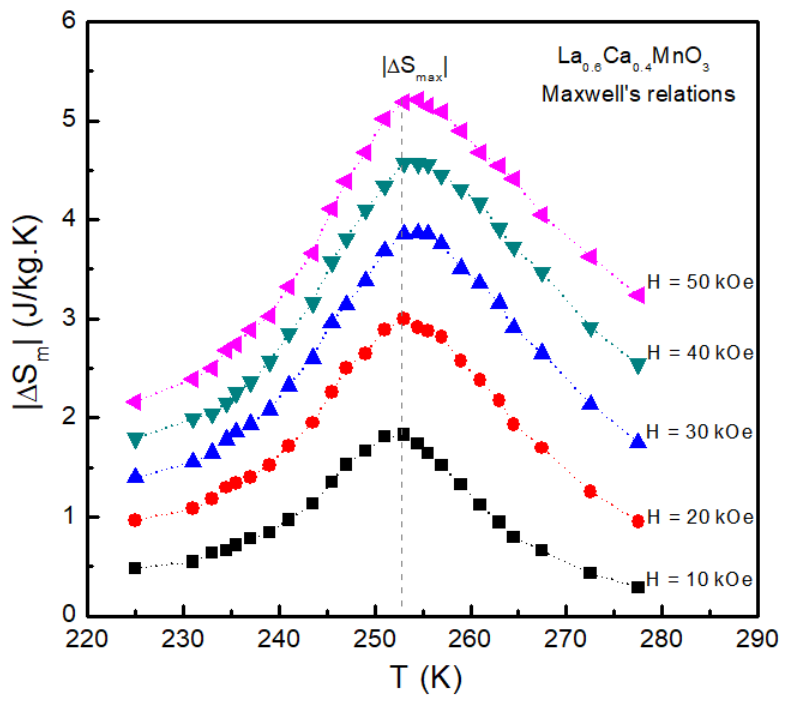

Fig. 3. (Color online) $\left|\Delta S_{m}(T)\right|$ data of LCMO for magnetic-field variations $H=10--50 \mathrm{kOe}$ calculated from Maxwell's relations. $\left|\Delta S_{\max }\right|$ is obtained around the $T_{C}$ point of the sample.

isotherms near $T_{C}$ with small temperature increments $(\Delta T)$. This also allows obtaining an accurate value of the maximum magnetic-entropy change $\left(\left|\Delta S_{\max }\right|\right)$, which is observed around the phase transition point, $T_{C}$.

For demonstration, Fig. 3 shows typical $\left|\Delta S_{m}(T)\right|$ data of LCMO for different $H$ variations from 10 to 50 kOe. These data were calculated by using Eq. (5) and isothermal $M(H)$ data. In general, $\left|\Delta S_{m}(T)\right|$ increases with increasing $H$. The largest increase in $\left|\Delta S_{m}\right|$ takes place around $\mathrm{TC} \approx 251 \mathrm{~K}$, corresponding to $\left|\Delta S_{\max }\right|$. An increase (or decrease) of temperature above (or below) $T_{C}$ leads to the reduction of $\left|\Delta S_{m}\right|$ due to changed magnetic order. Depending on magnetic order (long-range and/or short-range order) of an investigated sample, the peak position of $\left|\Delta S_{\max }\right|$ can be shifted (or un-shifted) towards higher temperatures when $\mathrm{H}$ increases. Because Maxwell's relations are widely used to calculate $\left|\Delta S_{m}\right|$ and give a high accuracy, the results obtained from the below methods will be compared with those obtained from the method using Maxwell's relations.

\section{2. $\Delta S_{m}$ calculation using Landau theory}

Landau theory is an effective theory used to describe phase-transition phenomena taking place around the critical point $T_{C}$ [43]. Landau considered the symmetry 
of a phase transition. For a magnetic system, it will be isotropic in the PM state. When it is in a FM state, however, the establishment of spontaneous magnetization breaks the rotation symmetry of the system and leads to anisotropic behaviour. The transition between two states with different symmetries is discontinuous since a given symmetry either exists or does not, characteristic of a FOPT. For a SOPT, the transition should separate two states with different symmetries. This defines an order parameter that fully describes the state of the system and its phase transition. According to Landau, any parameter that is null in the symmetric state and non-null in the non-symmetric state can be an order parameter [42]. For such magnetic system, the Gibbs free energy related to the magnetic energy is given by:

$$
G(M, T)=G_{0}+\frac{a}{2} M^{2}+\frac{b}{4} M^{4}+\frac{c}{6} M^{6}+\cdots-M H
$$

where $a, b$ and $c$ are temperature-dependent expansion coefficients containing the magnetoelastic coupling and electron condensation energy [41]. At $T_{C}$, the system reaches equilibrium condition, Gibbs free energy is minimum $(\partial G / \partial M=0)$, and the magnetic state equation can be expressed as:

$$
H=a M+b M^{3}+c M^{5}
$$

Here, the values of temperature-dependent $a, b$ and $c$ parameters are obtained by fitting experimental $M(H)$ data to Eq. (8), as shown in Fig. 4 for the case of LCMO. Among these parameters, $a(T)$ is always positive $[42,44]$, and $b(T)$ plays an important role in determining $\left|\Delta S_{m}\right|$ as well as the nature of a magnetic phase transition. The magnetic transition is first order if $b\left(T_{C}\right)$ is negative, otherwise it is second order. Particularly, in the phase-transition region, if $\mathrm{b}$ changes from a negative to positive value, the material exhibits the crossover behavior of the FOPT and SOPT, tricritical-like behavior [39]. Alternatively, it can be checked the nature of a phase transition by plotting $\mathrm{H} / \mathrm{M}$ versus $\mathrm{M} 2$ curves, Arrott plot [45]. A positive or negative slope of these curves means the SOPT or FOPT, respectively, as suggested by Banerjee criterion [46].

After collecting $a(T), b(T)$ and $c(T)$ values, and assessing the phase-transition type of the investigated material

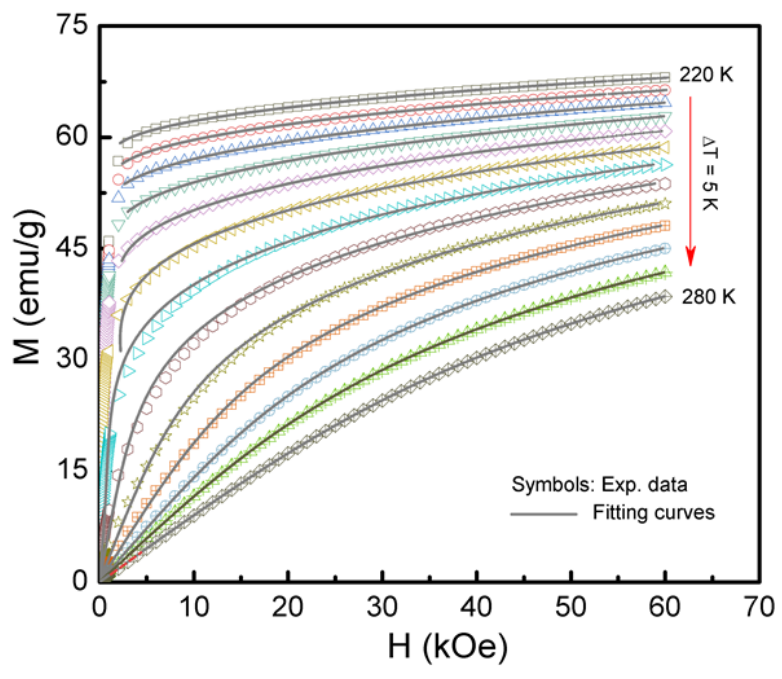

Fig. 4. (Color online) Representative $M(H)$ data of LCMO fitted to Eq. (8) deduced from Landau theory.

based on the sign of $b\left(T_{C}\right),\left|\Delta S_{m}\right|$ can be obtained from the Gibbs free energy relation as follows:

$$
\left|\Delta S_{m}(T, H)\right|=\frac{a^{\prime}}{2} M^{2}+\frac{b^{\prime}}{4} M^{4}+\frac{c^{\prime}}{6} M^{6}
$$

where $a^{\prime}, b^{\prime}$ and $c^{\prime}$ are the temperature derivatives of the expansion coefficients $a(T), b(T)$ and $c(T)$, respectively, which have been derived from fitting $M(H)$ isotherms, meaning Eq. (8) and Fig. 4. Because the magnetoelastic coupling and electron interactions can contribute to the magnetic entropy and its temperature dependence, these influence the shape of a $\left|\Delta S_{m}\right|(T)$ curve [41]. Fig. 5 shows $\left|\Delta S_{m}(T, H)\right|$ data of LCMO calculated from Landau theory in comparison to those obtained from Maxwell's relations. At a low field of $H$ $=10 \mathrm{kOe}$, one can see a significant difference in the $\left|\Delta S_{m}(T)\right|$ value around $T_{C}$. At higher fields, however, a good match between two data sets is observed. These results reflects that Landau theory is more suitable for the $\left|\Delta S_{m}(T)\right|$ calculation at high fields, where magnetic moments become saturated. At low fields, the demagnetization field, domain wall movement, and magnetocrystalline anisotropy can result in a deviation between the theoretical and experimental data. This can be seen from the fitting results of $M(H)$ data with $H<10 \mathrm{kOe}$ shown in Fig. 4. Additionally, Landau theory does not reflect the influence of the Jahn-Teller effect and exchange interactions, which could also be one of the reasons for the difference between $\left|\Delta S_{m}(T)\right|$ values obtained from two different methods. 


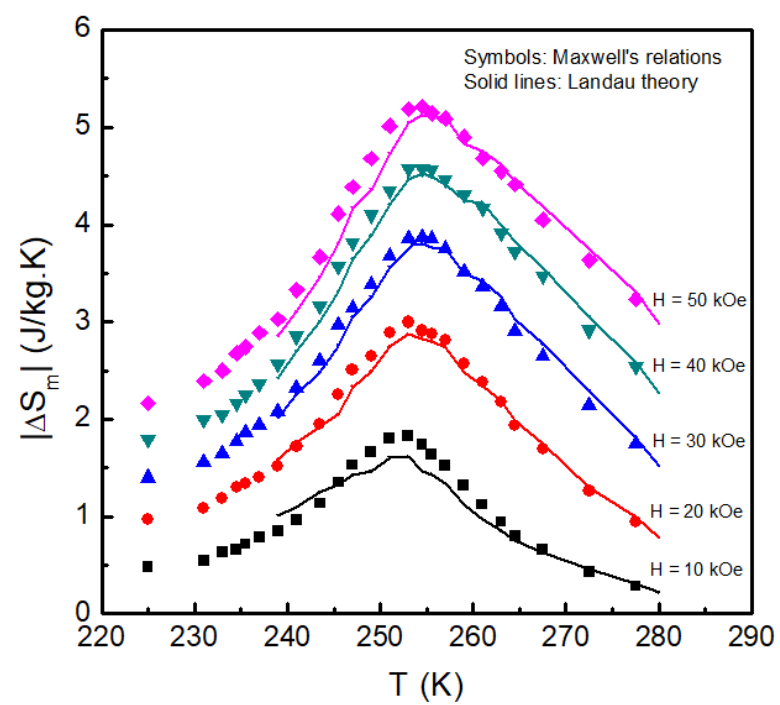

Fig. 5. (Color online) $\left|\Delta S_{m}(T)\right|$ data of LCMO with $H=10-50 \mathrm{kOe}$ calculated from Maxwell's relations (symbols) and Landau theory (solid lines), which are plotted in the same scale for comparison.

\section{3. $\Delta S_{m}$ calculation using classical PM theory}

\section{(Langevin function)}

In the classical limit, $M(T, H)$ of a paramagnet (a system of noninteracting magnetic moments) is described by the Langevin function [47]:

$$
M=\mu N\left[\operatorname{coth}(x)-\frac{1}{x}\right]
$$

where $x=\mu H / k_{B} T, \mu$ is atomic magnetic moment, $N$ is the number of noninteracting magnetic moments in the system, and $k_{B}$ is the Boltzmann constant. If a system consists of superparamagnetic nanoparticles, $M(T, H)$ can be described by a modified Langevin function [48]:

$$
M=\mu N\left[\operatorname{coth}(x)-\frac{1}{x}\right]+\chi H
$$

where $\mu$ is the average magnetic moment of clusters (or cluster size), $N$ is the number of the spin-clusters, and $\chi$ is the field-forced susceptibility associated with intrinsic magnetization of the PM phase. Depending on the nature of each system, one of these two equations will used for next calculation steps. In this work, we shall consider a conventional PM system, meaning that $M(T, H)$ is expressed in Eq. (10). By substituting Eq. (10) into Eq. (5) and carrying out the integration, it is obtained

$$
\begin{aligned}
& \left|\Delta S_{m}\right|[49]: \\
& \left|\Delta S_{m}(T, H)\right|=N K_{B}\left[1-x \operatorname{coth}(x)+\ln \left(\frac{\sinh (x)}{x}\right)\right]
\end{aligned}
$$

At low fields or high temperatures (in the PM region), $M$ follows the Curie law and Eq. (12) reduces to the following expression:

$$
\left|\Delta S_{m}(T, H)\right|=\frac{N \mu^{2} H^{2}}{6 k_{B} T^{2}}
$$

There is a note from Eq. (13) that if $\mu$ is large (and $N$ is simultaneously small to keep the saturation magnetization, $M_{s}=N \mu$, unchanged), $\left|\Delta S_{m}\right|$ increases because of the squared dependence on $\mu$, but linear dependence with $N$. This happens for superparamagnets, where the atomic magnetic moments cluster. Under that situation, $\left|\Delta S_{m}\right|$ increases with increasing the cluster size in the low field regime. However, there is a limit to this enhancement. According to Eq. (12), at high fields or very low temperatures (when $M \rightarrow M_{s}$, corresponding to the FM phase), $\left|\Delta S_{m}\right|$ will decrease when $\mu$ is increased. In this region, $M$ is no longer proportional to $\mu^{2}$ because it approaches $M_{s}$ and the reduction of $N$ becomes important. From Eq. (12), there is a maximum value of $\left|\Delta S_{m}\right|$ at $x_{\max }=\mu H / k_{B} T \approx 3.5$. This reflects that for the maximum MCE, there is an optimum cluster size $(\mu)$ for any given $T$ and $H$ values [49].

Applying Eqs. (10) and (12) to the system of LCMO with its $M(H, T)$ data shown in Fig. 2(b), we would obtain the results presented in Figs. 6 and 7 . It appears from Fig. (6) that the Langevin function well describes the $M(H)$ data in the PM region, as expected by theory. Around the FM-PM transition, however, there is a large difference between the experimental data and theoretical curves. This is due to the fact that when $T$ increases to $T_{C}$, the coupling between magnetic moments in the FM phase becomes declined, leading to noninteracting magnetic moments characteristic for the PM phase. Thus, the material can be considered as a composite consisting of FM clusters confined in a PM substance. In this case, Eq. (11) should be used to fit $M(H)$ data.

If seeing Fig. 7, it comes to our attention that the $\left|\Delta S_{m}(T)\right|$ data in the PM region $\left(T \geq T_{C}\right)$ calculated by using Maxwell's relations and the Langevin function are 


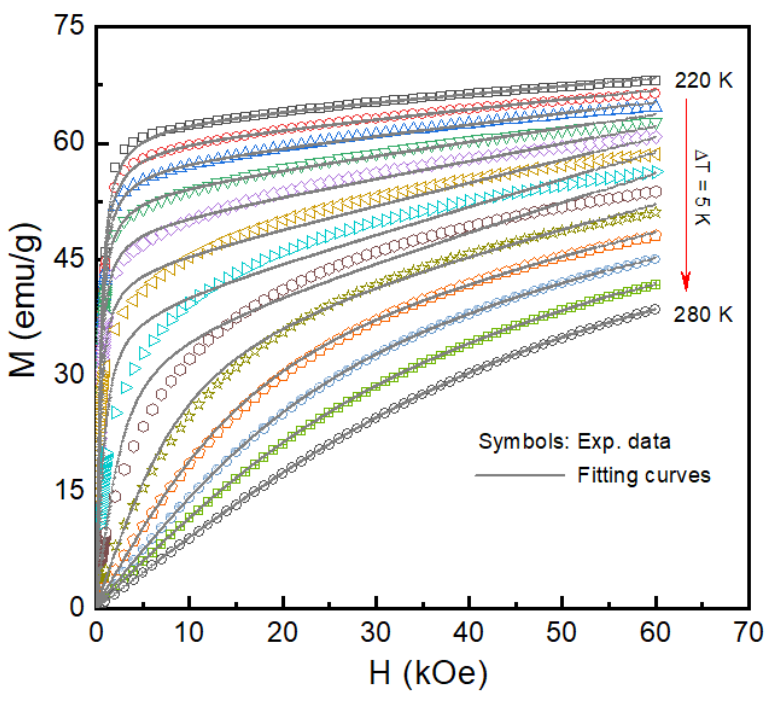

Fig. 6. (Color online) Representative $M(H)$ data of LCMO fitted to the Langevin function, Eq. (10).

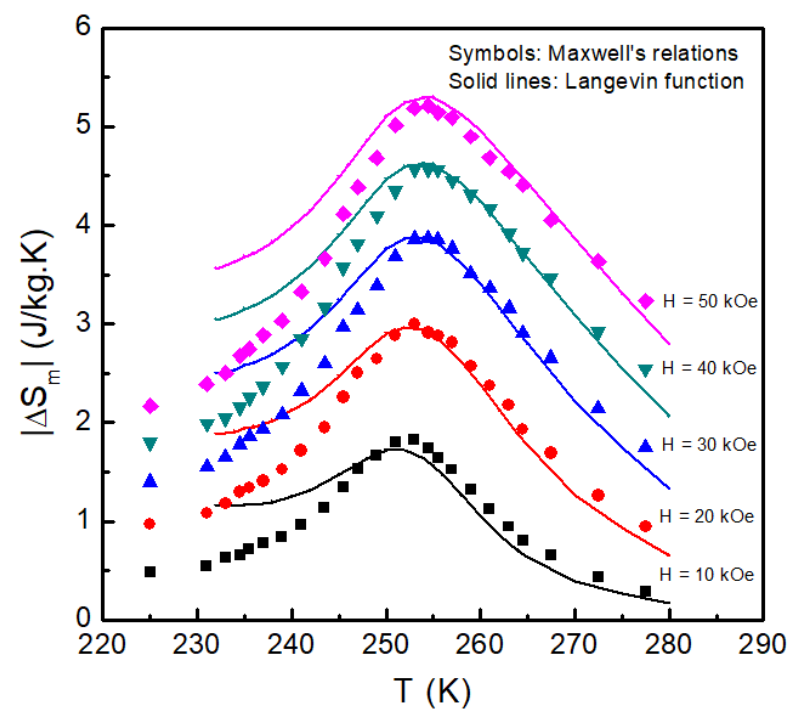

Fig. 7. (Color online) $\left|\Delta S_{m}(T)\right|$ data of LCMO with $H=10-50 \mathrm{kOe}$ calculated from Maxwell's relations (symbols) and the Langevin function (solid lines), which are plotted in the same scale for comparison.

fairly consistent with each other, excepting for the lowfield case of $H=10 \mathrm{kOe}$. The use of Eq. (13) for lowmagnetic fields could reduce this difference. For the FM phase, there are large differences between the $\left|\Delta S_{m}(T)\right|$ data calculated by two methods. As mentioned above, the coupling of magnetic moments and the Jahn-Teller effect, which are not included in Eqs. (10) and (11), could cause this phenomenon.

\section{4. $\Delta S_{m}$ calculation using mean-field theory}

Mean-field theory introduced by Weiss has described the most relevant thermodynamic phenomena of magnetic materials [50]. According to this model, the field induced by an average atom in a ferromagnet is the sum of the external field $H$ and an effective (or internal) field $H_{E}$ that is called the molecular field. One crucial point in this assumption is that $H_{E}$ is proportional to $M$ according to the relation $H_{E}=\lambda M$, where an effective-field factor $(\lambda)$ may depend on $M$ and/or $T$. It means that the total field of the system is given by $H_{\text {tot }}=H+H_{E}$. According to Amaral et al. [51], $\mathrm{M}$ in mean-field theory could be generalized as:

$$
M(T, H)=B_{s}\left[\left(H+H_{E}\right) / T\right]
$$

where $B_{s}$ is the Brillouin function associated with $M(T, H)$, which can be expressed as:

$$
\begin{gathered}
B_{s}(x)=\frac{2 J+1}{2 J} \operatorname{coth}\left(\frac{2 J+1}{2 J} x\right)-\frac{1}{2 J} \operatorname{coth}\left(\frac{x}{2 J}\right) \\
x=\frac{J g \mu_{B}}{k_{B}}\left(\frac{H+H_{E}}{T}\right)
\end{gathered}
$$

where $J$ is the total angular momentum, $g$ is the Landé factor, and $\mu_{B}$ is the Bohr magnetron. Taking the reciprocal function $B_{s}^{-1}(M)$, it is obtained the following relation:

$$
\frac{H}{T}=B_{s}^{-1}(M)-\frac{H_{E}}{T}
$$

Using $M(T, H)$ data, it will be presented $H / T$ versus $1 / T$ data corresponding to constant values of $M$, as shown in Fig. 8(a). These data are carried out the linear fitting to Eq. (17) in order to determine the best-fit values of $H_{E}$. To find $\lambda$, it is necessary to perform $H_{E}$ versus $M$ data, as shown in Fig. 8(b). According to the magnetic state equation as presented above, Eq. (8), the relation between $M$ and $H_{E}$ can be written as a polynomial function:

$$
H_{E}=\lambda_{1} M+\lambda_{3} M^{3}
$$

Using this equation, the fitting of the $H_{E}(M)$ data gives the values of $\lambda_{1}$ and $\lambda_{3}$. It has been found that $\lambda_{3}$ is very small as comparing with $\lambda_{1}$, and negative for 

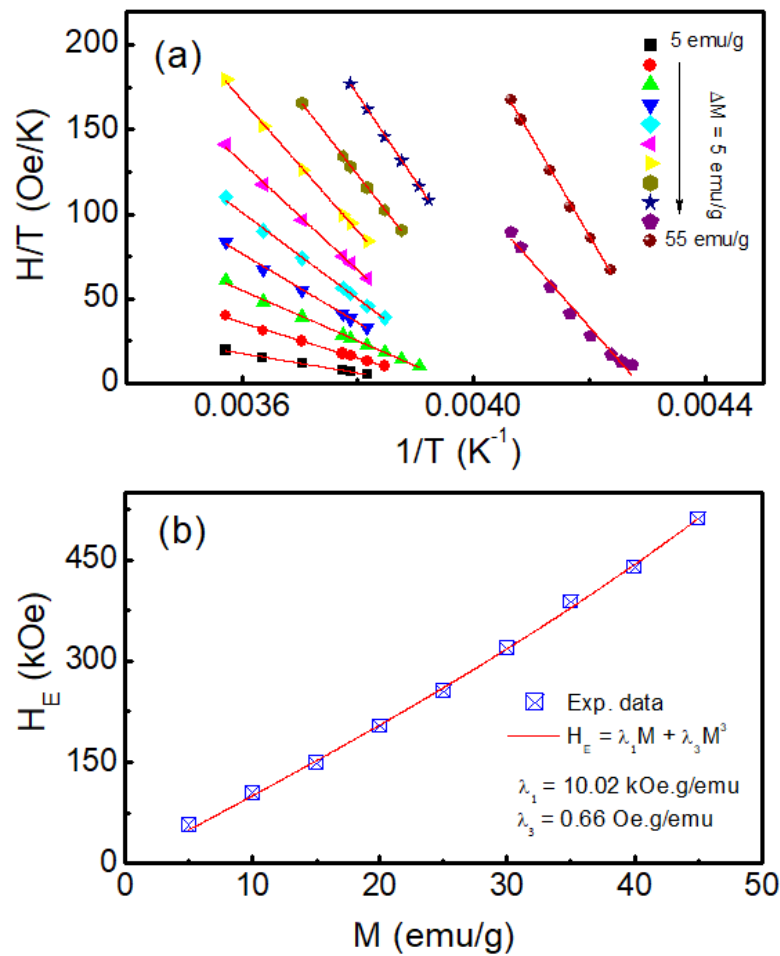

Fig. 8. (Color online) (a) $H / T$ versus $1 / T$ plots with constant values of $M$, and (b) $H_{E}$ versus $M$ data are fitted to Eq. (18); these data were corrected from LCMO.

the SOPT [36,51]. $H_{E}$ can thus be assigned to be equal to $\lambda_{1} M$, meaning $\lambda=\lambda_{1}$. For the case of LCMO, as shown in Fig. 8(b), the values of $\lambda_{1}$ and $\lambda_{3}$ are about $10.02 \mathrm{kOe} \cdot \mathrm{g} / \mathrm{emu}$ and $0.66 \mathrm{Oe} \cdot \mathrm{g} / \mathrm{emu}$, respectively. A positive value obtained for $\lambda_{3}$ is due to the fact that this material exhibits tricritical behavior (i.e., the crossover of the FOPT and SOPT) [39].

After obtaining the values of $H_{E}$ and $\lambda$, the next step is to build the scaling plot of $M$ versus $(H+H E) / T$ to determine $J$. With this plot, all $M(H)$ data collapse into a universal curve, as shown in Fig. 9(a). Using Eq. (17) to fit these data, it will be found the values of $J$ and $M_{s}$ as a function of temperature. Within the mean-field approach, $\left|\Delta S_{m}(T)\right|$ for a field variation from $H_{1}$ to $H_{2}$ can be calculated by using a general expression [51]:

$$
\left|\Delta S_{m}(T)\right|=\int_{M / H_{1}}^{M / H_{2}}\left[B_{s}^{-1}(M)-\left(\frac{\partial \lambda}{\partial T}\right)_{M} M\right] d M
$$

This equation also takes into account for a possible $\lambda(T)$ dependence. Normally, only the first term of the integral needs to be considered. In Fig. 9(b), it shows the $\left|\Delta S_{m}(T)\right|$ data of LCMO calculated by using Eq. (19) of mean-field theory, which are compared
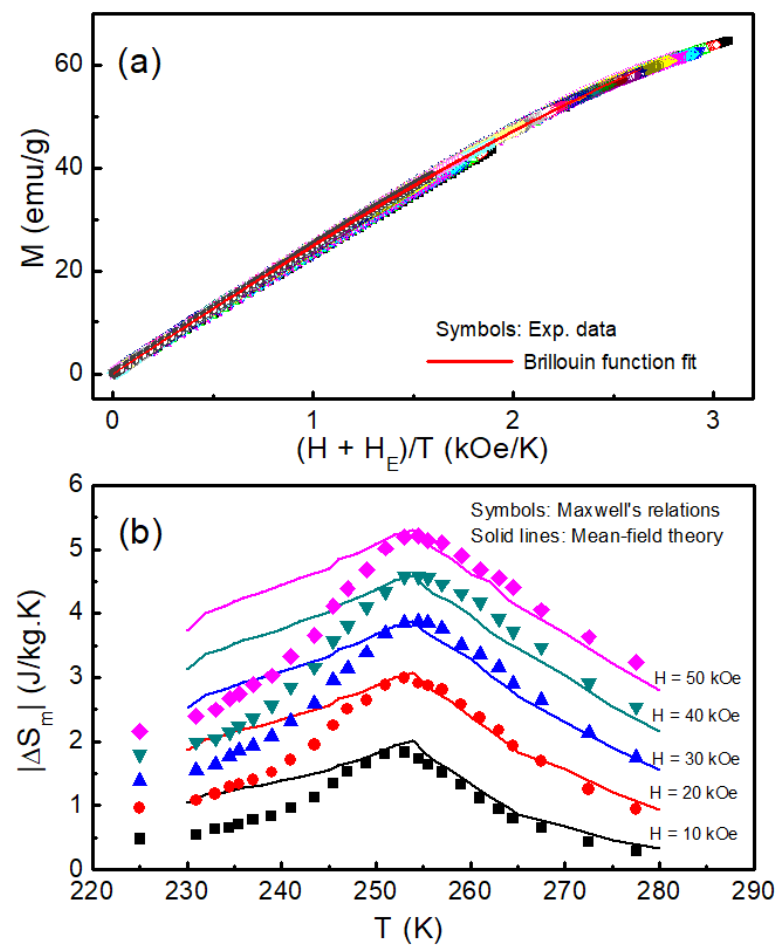

Fig. 9. (Color online) (a) Scaling plots of $\mathrm{M}$ versus $\left(H+H_{E}\right) / T$, and (b) $\left|\Delta S_{m}(T)\right|$ data of LCMO calculated from Maxwell's relations (symbols) and mean-field approximation (solid lines).

with those obtained by Maxwell's relations. Both data sets are fairly in agreement with each other at temperatures $T>T_{C}$. However, at temperatures $T<T_{C}$, a large difference could be due to the formation of magnetic domains with random orientation demagnetizationfield effect, and magnetocrystalline anisotropy that are not included in mean-field theory. Additionally, LCMO exhibits the crossover behavior of the FOPT and SOPT [39]. In good agreement with the results reported by Amaral and co-workers [51], who also found that the phase-transition nature and critical behaviors caused a difference between the $\left|\Delta S_{m}(T)\right|$ data calculated by different methods.

With the above presentations related to the case of LCMO, it comes to our attention that the calculation of $\left|\Delta S_{m}(T)\right|$ using Maxwell's relations gives the best results. Following this method, it is the use of Landau's phase-transition theory. This method is really effective if applying to MCE materials undergoing the SOPT. The use of Langevin function (classical PM theory) and mean-field theory can give anomalous $\left|\Delta S_{m}(T)\right|$ data that are inconsistent with those obtained from two above 
methods at temperatures $T<T_{C}$, particularly for materials exhibiting the FOPT [51] or crossover behavior (such as the case of LCMO). The deviation between the experimental data and theoretical curves could be due to the fact that Langevin function and mean-field theory do not include the terms related to magnetic domains with different orientations, demagnetization-field effect, magnetocrystalline anisotropy, Jahn-Teller effect, and/or magnetostructural/magnetoelastic coupling. A full model including all these terms is expected to overcome the above problems.

\section{Figures of merit of a MC material}

Apart from assessing two parameters $\Delta T_{a d}$ and $\left|\Delta S_{m}\right|$, it is necessary to additionally evaluate $R C P$ and $R C$, which are known as figures of merit of a MC material. From the $\left|\Delta S_{m}(T)\right|$ data determined for a given $H$ variation, $R C P$ and $R C$ are defined as follows $[2,10]$ :

$$
R C P=\left|\Delta S_{\max }\right| \times \delta T
$$

$$
R C=\int_{T_{1}}^{T_{2}}\left|\Delta S_{m}(T)\right| d T
$$

In Eq. (20), $\delta T$ is the linewidth or the full width at half maximum (FWHM) of a $\left|\Delta S_{m}(T)\right|$ curve, as demonstrated in Fig. 10(a). Meanwhile, $T_{1}$ and $T_{2}$ in Eq. (21) are the cold and hot ends, respectively, of an ideal thermodynamic cycle. Basically, $T_{1}$ and $T_{2}$ can be selected at the $\frac{1}{2}\left|\Delta S_{\max }\right|$ value of a $\left|\Delta S_{m}(T)\right|$ curve, and thus $R C$ is the integrated area between $T_{1}$ and $T_{2}$, as shown in Fig. 10(b).

In practice, in a working temperature range, it is expected to achieve $R C P$ (or $R C$ ) of a MC material as large as possible. In other words, a large $R C P$ for the same $H$ value indicates a better MC material [10]. In general, both $R C P$ and $R C$ increase with increasing $\mathrm{H}$, due to the increase of $\left|\Delta S_{\max }\right|$ and $\delta T$ at high fields. This can be clear seen in Fig. 11 and its inset for the case of LCMO. It is worth noting that at any field, $R C P$ is always larger than $R C$. The ratio of $R C P / R C$ also increases with increasing $H$. Further analysis of $H$-dependent $R C P, R C$ and $\delta T$ data, it has been found they can be described by
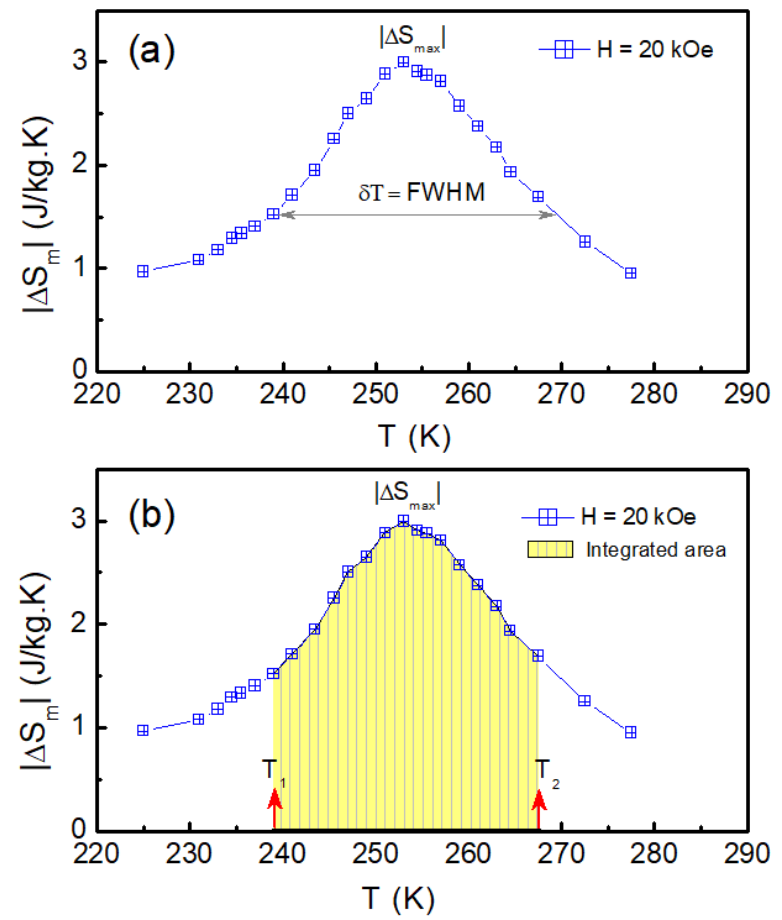

Fig. 10. (Color online) Definition of some MC parameters related to a $\left|\Delta S_{m}(T)\right|$ curve that are used to calculate (a) $R C P$ and (b) $R C$. Here, the $\left|\Delta S_{m}(T)\right|$ data of LCMO for $H=20 \mathrm{kOe}$ calculated by using Maxwell's relations are plotted for illustration.

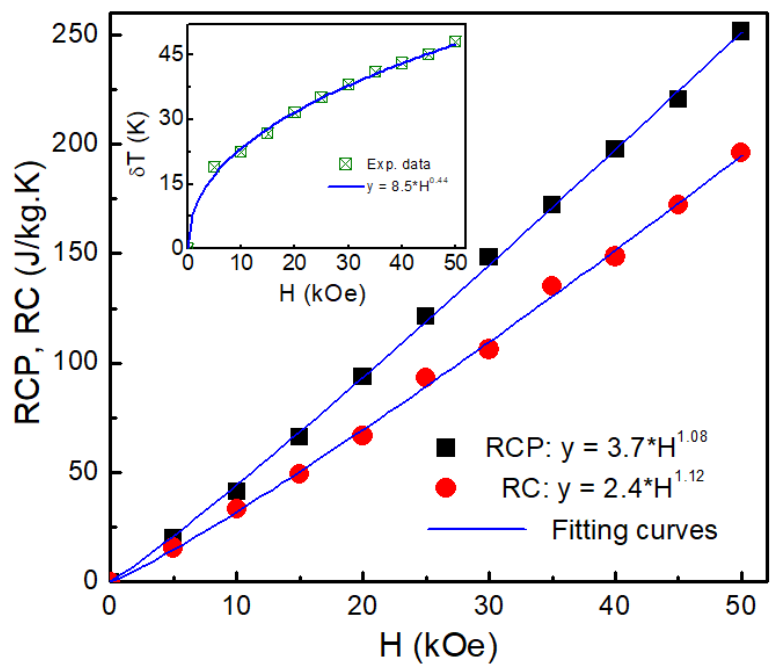

Fig. 11. (Color online) $R C P(H), R C(H)$ and $\delta T(H)$ data of LCMO are fitted to a power law $y \propto H^{n}$.

a power function of $y \propto H^{n}$, where $n$ is the exponent, as shown in Fig. 11. If combining with the results obtained from analyzing $\left|\Delta S_{m}(H)\right|$ and $\left|\Delta S_{\max }(H)\right|$ data, the consideration of $n$ can get more information about magnetic order [2]. 


\section{Conclusion}

We reviewed the typical methods using Maxwell's relations, and Landau, classical PM and mean-filed theories to calculate $\left|\Delta S_{m}\right|$ from $M(H)$ isotherms of LCMO. The reviewing has exhibited that the methods using Maxwell's relations and Landau theory are easy to apply and give $\left|\Delta S_{m}\right|$ values in good agreement with each other. Comparing with these methods, two other methods (using the classical PM and mean-filed theories) can cause a large deviation, particularly at temperatures $T<T_{C}$. The presence of many parameters (such as $J, g, \mu, \lambda$, and $\left.H_{E}\right)$ in the expressions, that their value needs to be approximately calculated, is thought to cause the above phenomenon. Additionally, the classical PM and mean-field theories do not include the terms related to magnetic domains with different orientations, demagnetization field, Jahn-Teller effect, and/or magnetostructural/magnetoelastic coupling. The lack of these terms also influences the results of the $\left|\Delta S_{m}\right|$ calculation. In this work, we also presented the calculation methods of $R C P$ and $R C$, where their dependence on $H$ is assessed in comparison with each other.

\section{ACKNOWLEDGEMENTS}

The research at Korea was supported by the National Research Foundation of Korea Grant No. 2020R1A2C1008115.

\section{REFERENCES}

[1] A. M. Tishin and Y. I. Spichkin, The magnetocaloric effect and its applications (IOP Publishing Ltd, Bristol and Philadelphia, 2003).

[2] V. Franco et al., Mater. Sci. 93, 112 (2018).

[3] O. Sari and M. Balli, Int. J. Refrig. 37, 8 (2014).

[4] J. Glanz, Science 279, 2045 (1998).

[5] V. Franco, J. S. Blazquez, B. Ingale and A. Conde, Annu. Rev. Mater. Res. 42, 305 (2012).

[6] W. Zhong, C. T. Au and Y. W. Du, Chinese Phys. B 22, 057501 (2013).

[7] S. Gorsse, B. Chevalier and G. Orveillon, Appl. Phys. Lett. 92, 122501 (2008).
[8] S. Y. Dan'kov, A. M. Tishin, V. K. Pecharsky and K. A. Gschneidner Jr., Phys. Rev. B 57, 3478 (1998).

[9] Y. S. Koshkid'ko et al., J. Magn. Magn. Mater. 433, 234 (2017).

[10] K. A. Gschneidner Jr. and V. K. Pecharsky, Annu. Rev. Mater. Sci. 30, 387 (2000).

[11] K. A. Gschneidner Jr, V. K. Pecharsky and A. O. Tsokol, Rep. Prog. Phys. 68, 1479 (2005).

[12] V. K. Pecharsky and K. A. Gschneidner Jr., Phys. Rev. Lett. 78, 4494 (1997).

[13] V. K. Pecharsky and K. A. Gschneidner Jr., Adv. Mater. 13, 683 (2001).

[14] Y. F. Chen et al., J. Phys.: Condens. Matter 15, L161 (2003).

[15] S. Fujieda, A. Fujita and K. Fukamichi, Appl. Phys. Lett. 81, 1276 (2002).

[16] O. Gutfleisch, A. Yan and K.-H. Müller, J. Appl. Phys. 97, 10M305 (2005).

[17] W. Z. Nan et al., New Phys.: Sae Mulli 67, 812 (2017).

[18] M. P. Annaorazoy and S. A. Nikitin, J. Appl. Phys. 79, 1689 (1996)

[19] T. L. Phan et al., Appl. Phys. Lett. 101, 212403 (2012).

[20] V. Basso et al., Phys. Rev. B 85, 014430 (2012).

[21] T. Krenke et al., Nat. Mater. 4, 450 (2005).

[22] X. Zhou, W. Li, H. P. Kunkel and G. Williams, J. Phys.: Condens. Matter 16, L39 (2004).

[23] X. Zhang et al., J. Alloys Compd. 656, 154 (2016).

[24] X. Moya et al., Nat. Mater. 12, 52 (2013).

[25] T. L. Phan et al., J. Appl. Phys. 112, 093906 (2012).

[26] H. B. Li et al., Mater. Chem. Phys. 107, 377 (2008).

[27] A. Rebello, V. B. Naik and R. Mahendiran, J. Appl. Phys. 110, 013906 (2011)

[28] Y. D. Zhang et al., New Phys.: Sae Mulli 61, 915 (2011).

[29] T. L. Phan, J. Korean Phys. Soc. 61, 429 (2012).

[30] S. Y. Dan'kov, A. M. Tishin, V. K. Pecharsky and K. A. Gschneidner Jr., Phys. Rev. B 57, 3478 (1998).

[31] P. T. Phong et al., J. Alloys Compd. 683, 67 (2016).

[32] M. Jeddi et al., RSC Adv. 8, 9430 (2018).

[33] C. M. Xiong et al., IEEE Trans. Magn. 41, 122 (2005). 
[34] N. Y. Pankratov, V. I. Mitsiuk, V. M. Ryzhkovskii and S. A. Nikitin, J. Magn. Magn. Mater. 470, 46 (2019).

[35] M. Triki et al., 509, 9460 (2011).

[36] A. Belkahla et al., Appl. Phys. A 125, 443 (2019).

[37] R. D. McMichael, J. J. Ritter and R. D. Shull, J. Appl. Phys. 73, 6946 (1993).

[38] M. A. Hamad, Phase Transit. 85, 106 (2012).

[39] P. Zhang et al., J. Magn. Magn. Mater. 348, 146 (2013).

[40] Y. D. Zhang, T. L. Phan and S. C. Yu, J. Appl. Phys. 111, 07D703 (2012).

[41] J. S. Amaral et al., J. Magn. Magn. Mater. 290, 686 (2005).

[42] A. Bouderbala, J et al., Ceram. Int. 41, 7337 (2015).

[43] H. E. Stanley, Introduction to phase transitions and critical phenomena (Oxford University Press, London, 1971).
[44] M. S. Anwar et al., J. Korean Phys. Soc. 60, 1587 (2012).

[45] A. Arrott, Phys. Rev. 108, 1394 (1957).

[46] B. K. Banerjee, Phys. Lett. 12, 16 (1964).

[47] B. D. Cullity, Introduction to Magnetic Materials (Addison-Wesley Publ. Co., Reading, MA, 1974).

[48] R. Saha, V. Srinivas and A. Venimadhav, J. Magn. Magn. Mater. 324, 1296 (2012).

[49] R. D. Shull, R. D. McMichael, L. J. Swartzendruber and L. H. Bennett, edited by J. L. Dormann and D. Fiorani (Elsevier Science Publishers B.V., 1992) p. 161.

[50] P. Weiss, J. Phys. Theor. Appl. 6, 661 (1907).

[51] J. Amaral, N. Silva and V. Amaral, Appl. Phys. Lett. 91, 172503 (2007). 